\title{
Dynamics and Electrooptics of Vertically Aligned Nematics With Induced Pretilt on $\mathrm{SiO}_{x}$
}

\author{
Eva Otón, Sol López-Andrés, Carlos Carrasco-Vela, Beatriz Cerrolaza, Noureddine Bennis, and José Manuel Otón
}

\begin{abstract}
The response time and contrast of vertically-aligned negative nematics depend critically on the aligning surface and its induced pretilt. Pretilt can be tuned up using obliquely evaporated inorganic oxides as alignment surface.

A thorough study of pretilt angles induced by aligning surfaces of thermally evaporated $\mathrm{SiO}_{x}$ has been carried out on several commercial and experimental negative nematic mixtures. Morphology of $\mathrm{SiO}_{x}$ with different evaporation angles has been studied by Field Emission Scanning Electron Microscopy. Some morphological variations appear at specific evaporation angles. These are related to the pretilt induced by the surface and to the dynamic response of the device.
\end{abstract}

Index Terms-Field-emission scanning electron microscopy (FESEM), liquid crystals, $\mathrm{Si}_{x}$, vertically aligned nematic (VAN), vertical alignment.

\section{INTRODUCTION}

V ERTICALLY aligned nematic (VAN) liquid crystals with a negative dielectric anisotropy are useful in many applications requiring fast response time and excellent contrast ratio. VAN displays have become popular in consumer electronics because of their excellent contrast and low response time (often reaching video frequency). At present, a number of solutions based on VANs (MVA, PVA, etc.) can be found in commercial products, like large-area direct-view TV sets and high-end projection displays [1], [2]. VANs, on the other hand, are excellent candidates for a number of photonic devices-beamsteerers, tunable lenses, prisms, or filters - whose action on an impinging light beam is restricted to phase delays, i.e., without modifications of the light intensity or the state of polarization. Indeed, a VAN cell upon switching can be employed as an analog phase-only linear retarder i.e., produces arbitrary phase delays depending on voltage, without modifying the state of polarization of a linear incoming light.

In either case, the display performance is ultimately determined by its contrast (or visibility) and its dynamic response. Both parameters depend on the pretilt angle induced by the aligning surface on the liquid crystal molecules; therefore pretilt must be controlled [3]. Pure homeotropic alignment (pretilt angle $=\mathbf{0}^{\circ}$ ) would produce theoretically a contrast only limited by the light leakage of the polarizers. However is useless for actual devices since the switching torque is null (in practice, switching time is exceedingly long), and the switching direction is undefined. Therefore, a non-null induced pretilt angle is require for the material to switch along a predefined direction. As pretilt is increased, the response time improves but contrast ratio is compromised.

It has been shown that pure vertically alignment can be obtaine by doping the host liquid crystal with other materials [4]. However, pretilt angles cannot be controlled with this method. Recently, vertically alignments have been achieved by the use of ion and plasma beams [5], as well as depositions by a filtered cathodic arc process, leading to a different variation of pretilt angles [6].

In this work, the aim has been to generate a controlled pretilt angle depending on the evaporation angle and determine a relation between $\mathrm{SiO}_{x}$ surface structure and pretilt. The generation of pretilt angles using $\mathrm{SiO}_{x}$ as alignment layer has been studied. $\mathrm{SiO}_{x}$ layers were obtained by oblique PVD (Physical Vapor Deposition). $\mathrm{SiO}_{u:}$ surface morphology has been studied using scanning microscopy techmiques to find a relation between surface structure and liquid crystal pretilt angle. Different evaporation angles produce different pretilts or even different types of alignments. These pretilts have been measured along with their corresponding variations of contrast and dynamic response. Low pretilt angles, i.e., nearly homeotropic or vertical orientation, guarantee the best contrast ratios, due to perfect vertical alignment, but response time is remarkably longer. On the other hand, devices having high pretilt angles show excellent response times but poor contrast ratio. Therefore, pretilt needs to be tightly controlled in order to achieve a trade-off between good contrast ratio and fast response time [7].

\section{EXPERIMENTAL}

Two kinds of experiments have been done. Several batches of ITO-coate glasses with evaporate $\mathrm{SiO}_{x}$ at different angles were prepare for SEM microscopic analysis. The analysis occasionally damages the $\mathrm{SiO}_{x}$; therefore, it was decided not to use the same batches for cell manufacturing. Instead, parallel batches of every orientation and manufacturing condition were prepared. The second batch was employed for cell manufacturing. Single pixel cells were assembled in antiparallel orientation. The cell gap was $4 \mu \mathrm{m}$ in all cases. A nematic commercial mixture MLC 6608 (Merck) with a negative dielectric anisotropy was filled at isopic state and allowed to cool down following a standard manufacturing protocol. 


\section{A. Si $\boldsymbol{O}_{x}$ PVD (Physical Vapor Deposition)}

Several evaporations have been done using PVD techmique. Selected angles covere a range from $20^{\circ}$ to $86^{\circ}$. Each evaporation for every group of samples consisted of: first, deposition of $100 \AA$

speed of $1 \AA / \mathrm{s}$, then, selecting the desired angle, evaporation at $1 \mathrm{~A} / \mathrm{s}$ and $100 \mathrm{~A}$ thick. It must be mentioned that our evaporation chamber features several modifications including an external goniometer actuator attached to the sample. Therefore, the orientation of the batch can be modified and the second evaporation can be performed without breaking the vacuum.

$\mathrm{SiO}_{x}$ surface structures were analyzed by FESEM (Field Emission Scanning Electron Microscopy), starting at $20^{\circ}$ and studying surface variations in each sample with a difference of $10^{\circ}$. Zoom had to be set at $x 60000$ due to the extremely high uniformity and flatness of the surfaces.

\section{B. Pretilt Measurements}

Pretilt measurements have been performed in the whole series of manufactured cells with $\mathrm{SiO}_{x}$ layers deposite at different evaporation angles. Pretilt angles were measured using an ellipsometric method described elsewhere. The ellipsometric system is able to detect minute changes in the state of polarization (SOP) of the incoming light, thus resulting very sensitive for pretilt measurements. The ellipsometer actually measures the tilt angle of the liquid crystal molecules across the cell. Measurements are indirect, i.e., the tilt is obtained as the best fit of the liquid crystal profile producing a given set of SOP variations. The metho is quite reliable since ata acquisition is performed at several impinging angles and wavelengths. A full discussion of the method can be found in [8].

Within this experimental context, pretilt is simply the tilt of the liquid crystal adjacent to the glass surfaces. The precision can be further improved by repeating the procedure for several cells of every evaporation angle. Pretilt is referre to the surface normal, i.e., $0^{\circ}$ means perpendicular and $90^{\circ}$ means parallel to the glass surface.

\section{Electrooptic Characterization}

Quasi-static and dynamic electrooptical characterization of the cells was carried out by recording the time resolved optical transmission of the samples between crossed polarizers while being addressing with driving voltage signals. Cells are placed in a microscope (Nikon Optiphot Pol 2) provided with a programmable hot plate and a rotating stage. All cells were measured at $35^{\circ} \mathrm{C}$. The output light is collected in an optical fiber bunch bringing the signal to a fast response photodiode (Hamamatsu). The photodiode signal is store in a digital oscilloscope (Hewlett Packard) and sent to a computer. The same computer controls the electronic driving of the cell via a LabView application. Cells were addressed using a $10 \mathrm{kHz}$ AC carrier modulated by a $1 \mathrm{~Hz}$ square signal with variable amplitude.

\section{RESULTS AND DISCUSSION}

\section{A. Si $\boldsymbol{O}_{x}$ Surface Structure-Ceneral View From $20^{\circ}$ to $86^{\circ}$}

Observed surfaces correspond to $20^{\bullet}, 30^{\bullet}, 40^{\bullet}, 50^{\bullet}, 60^{\bullet}, 70^{\bullet}$, $80^{\circ}, 82^{\circ}, 84^{\circ}, 85^{\circ}$, and $86^{\circ}$, see Fig. 1.
From $20^{\circ}$ to $40^{\circ}$, the uniformity of the surface morphology is very high, with little or no features. Non-polyhedral particles are found; morphology appears to be a 'droplet-like' structure. Droplets are rounded shaped though not regular, with no apparent orientation and placed randomly. Droplet diameter is variable between 100 and $250 \mathrm{~nm}$ approximately. Generally speaking, the particle size increases as evaporation angle increases.

From $50^{\circ}$ to $80^{\circ}$, surface structures show some variations. Particles or droplets become more defined, with sharper delineated boundaries. They also have a more rounded shape and their size appears to be larger than for lower angles. Near $80^{\circ}$, surface structure starts being less uniform having little holes or cracks between particles.

A sudden change in morphology occurs for angles greater than $80^{\circ}$. A more detailed study was done for samples with evaporation angles from $80^{\circ}$ to $86^{\circ}$, as its morphology showed a remarkable alteration: structure and particle size are different, and particle distribution highly differs. In this case surface was less uniform than the surface observed at lower evaporation angles, showing irregularities along the whole sample and a more detailed structure. The particle size radically changes, showing uniformity and diameters from 30 to $50 \mathrm{~nm}$.

In these cases, a progressive evolution of the surface structure was observed, changing from 'droplet-like' structure to spherulitic morphology. Evaporations for $82^{\bullet}$ and $84^{\bullet}$ show intermediate morphology states, coexisting both droplet and spherulitic structures. $85^{\circ}$ and $86^{\circ}$ show, a spherulitic or a granulate morphology, with some spherical particles as well, with sharper edges, smaller particles and presence of holes at high angles. Holes have a triangular shape, according to a sphere stacking structure. Additionally, structure at higher angles is less compact, has bigger holes and spherical isolated particles are present. Alignment of particles and holes perpendicular to evaporation direction is observed. Thicker $\mathrm{SiO}_{x}$ layers lead to an oblique columnar structure [9].

\section{B. Liquid Crystal Alignment}

A series of liquid crystal cells were manufactured with $\mathrm{SiO}_{x}$ as alignment layer, changing evaporation angle in $10^{\circ}$ steps for non-variable alignments and in $2^{\bullet}$ when alignment showed a bigger variation. Table I shows the measure pretilt angle of the liquid crystal molecules depending on $\mathrm{SiO}_{x}$ evaporation angle.

The liquid crystal alignment strongly depends on the evaporation angle set on the outer glass plates of the cell. As a rough approximation, liquid crystal cannot be aligned vertically if evaporation angle is lower than $\mathbf{5 0}^{\circ}$. Vertical alignment shows up for evaporation angles greater than $50^{\circ}$.

Samples with evaporations from $20^{\circ}$ to $50^{\circ}$ present mostly an homogeneous alignment, clear state is present at $45^{\circ}$ between crossed polarizers.

Fig. 2 shows several cells observed between crossed polarizers. $20^{\circ}$ and $40^{\circ}$ cells show lack of aligmnent and scattering. As a rule, cells obtained with deposition angles lower than $50^{\circ}$ were useless; no vertical alignment can be obtained.

Alignment in samples with evaporations from $50^{\circ}$ to $80^{\circ}$ presents the most interesting results. $\mathrm{SiO}_{x}$ evaporated in this 

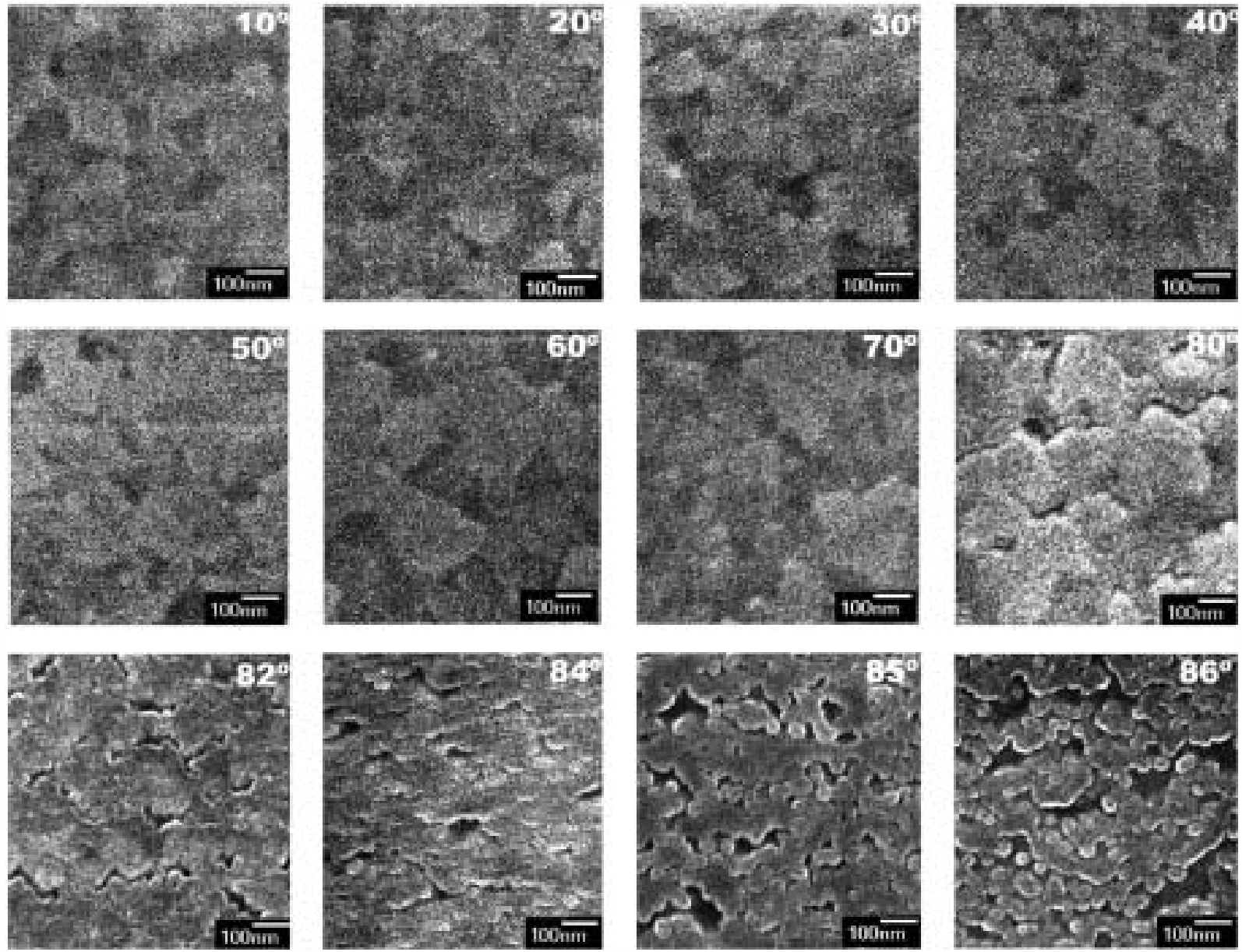

Fig. 1. $\mathrm{Si} \boldsymbol{\bullet}_{x}$ surface morphology, evaporation angles from $20^{\circ}$ to $86^{\circ}$ analysed by FESEM.

TABLE I

Liquid Crystal Pretilt ANGles ObTained for DifFerent Si $\boldsymbol{Q}_{x}$ EVAPORATION ANGLES

\begin{tabular}{|c|c|c|c|c|c|}
\hline Evaporation angle & $<50^{\circ}$ & $50^{\circ}-60^{\circ}$ & $70^{\circ}$ & $80^{\circ}$ & $>85^{\circ}$ \\
\hline LC pretilt angle & Random & $\sim 1^{\circ}$ & $\sim 8^{\circ}$ & $\sim 26^{\circ}$ & $\sim 90^{\circ}$ \\
\hline
\end{tabular}

range will produce vertically aligned cells and thus, useful devices. It is worth noticing that, as mentioned abøve, only minute variations in the $\mathrm{SiO}_{x}$ surface morphology are appreciated when comparing surfaces in the $20^{\circ}-50^{\circ}$ range and surfaces in the $50^{\circ}-\mathbf{8 0 ^ { \circ }}$. These minute variations scarcely visible at very high zoom give rise, however, to a dramatic change in the liquid crystal molecular orientation. It is reasonable to assume that increasing the evaporation angle, the anchoring strength of the surface increases as well. A method for measuring the anchoring strength by ellipsometry has been recently developed, and is currently being applied to this problem.

Cells that have been manufactured with glass plates in the $50^{\circ}-60^{\circ}$ range are black with no transmission variations when rotating the cell between crossed polarizers; this is a typical response of vertical aligned cells. Pretilt angle increases with evaporation angle; consequently, the higher the evaporation angle (from $50^{\circ}$ onwards), the higher the cell transmission. Cells in the $60^{\circ}-80^{\circ}$ range show a small light transmission when placing the cell at $45^{\circ}$ within the polarizers. This is a result of an increasing pretilt making the cell more and more birefringent as the evaporation angle increases.

Cells with evaporation angles at $50^{\circ}, 60^{\circ}, 70^{\circ}$ and $80^{\circ}$ are shøwn in Fig. 2. $50^{\circ}$ cell shows no transmission (black), therefore pretilt is null or near null, and cell will show a gøod contrast ratio. $60^{\circ}$ cells have the same appearance as $50^{\circ}$. Again, pretilt angle is null and there's no transmission. $50^{\circ}$ and $60^{\circ}$ liquid crystal alignments are equivalent being their pretilt angle close to zero. Ellipsometric measurements show that the pretilt angle is about $1^{\circ}$ at most.

Alignment $\bullet$ btained for $70^{\circ}$ cells is different. Hømeotropic alignment is achieved as well but a certain transmission in the cell can be seen because $70^{\circ}$ cells have a certain pretilt angle and contrast decreases. Pretilt angle as measured by spectral ellipsometry is about $8^{\circ}$.

$80^{\circ}$ cells exhibit the highest transmission. Pretilt angle increases considerably being close to $26^{\circ}$, which is too high for most applications requiring hometropic alignment. The contrast ratiø is consequently compromised.

Samples with evaporation angles from $80^{\circ}$ to $86^{\circ}$ showed interesting variations. As mentioned before, $\mathrm{SiO}_{x}$ morphølogy for angles greater than $80^{\circ}$ changes drastically from lower angles. Liquid crystal alignment manifests a variation in the same way. For high angles, pretilt angle is sø high that alignment cannot 


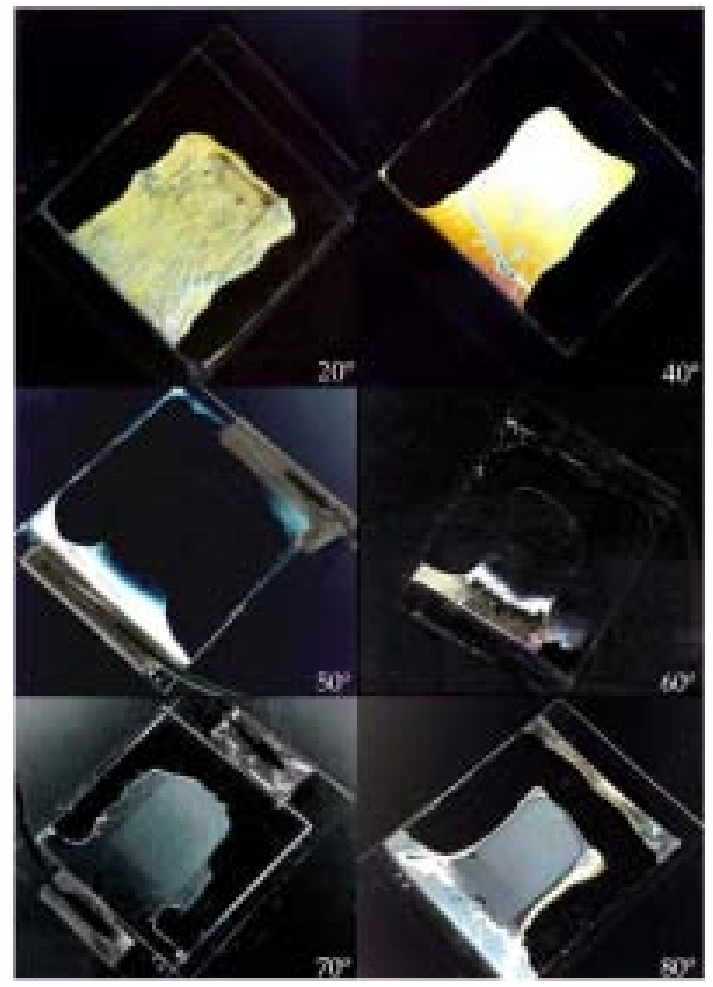

Fig. 2. Displays from $20^{\circ}$ to $80^{\circ} .20^{\circ}$ and $40^{\circ}$ evaporation angle show non aligned liquid crystal. $50^{\circ}, 60^{\circ}, 70^{\circ}$ and $80^{\circ}$ evaporation angle surfaces show vertical alignment of the LC, but pretilt increases, as seen by the increased transmission between crossed polarizers.

be considered homeotropic anymore. Evaporation angle of $85^{\circ}$ produces an almost homogeneous alignment, molecules having a pretilt close to $\mathbf{9 0}^{\circ}$, i.e., parallel to the glass plates. Contrast in these cells is very low, and switching is barely noticeable. Note that switching would intend to bring the molecules to any position within the glass plates lane. Since the molecules are already on this plane in steady state, no reorientation is required for the liquid crystal to "switch".

1) Modifying Evaporation Parameters: A group of cells with different evaporation parameters was analyzed to check alignment uniformity.

A number of evaporations were made to verify the PVD parameters. A batch with layer thickness $300 \AA$ was prepared to confirm that the $100 \AA$ layer thickness is thick enough to shield any effect from the vertically evaporated layer. Similarly, using a $\mathbf{0 , 2} \AA / \mathrm{s}$ evaporation speed confirmed that the same ranges of alignments as in $1.0 \AA / \mathrm{s}$ were obtained. Liquid crystal alignment did not show any variation with the new parameters. It is assumed, therefore, that within a certain range, evaporation angle is the only relevant manufacturing parameter affecting the pretilt in $\mathrm{SiO}_{x}$ aligning surfaces

\section{Electrooptic Characterization}

All the test cells included in each batch for every evaporation angle were characterized electrooptically in terms of contrast, response time and greyscale. As expected, pretilt angle has a strong influence in the liquid crystal cells, especially in contrast and response time. On average, low pretilt cells, corresponding to $50^{\circ}$ and $60^{\circ}$ evaporation angle have significantly higher contrast ratios but worse response times than cells having high pretilt angles as obtained with cells of $70^{\circ}$ and $80^{\circ}$ evaporation angles. The response time, as measured in the whole range of evaporation angles, was in the range of $2-3 \mathrm{~ms}$, while contrast steadily decreased when increasing evaporation angle.

1) Backflow Effect: The rise time of any LC display is usually shorter as the applied voltage increases, so liquid crystal molecules switch faster at higher voltages. In some of the manufactured cells the opposite behavior was found.

For displays having null or near null pretilt angle, the response time increases above a certain voltage value, and rise time achieve very long values. However, fall times are not affected. This effect alters the measurement of response times and is called backflow effect [10]

Above certain voltages, there is a fast rearrangement of the liquid crystal molecules followed by a slow reorientation, which induces the molecules to switch slowly increasing the overall rise time. From the practical point of view, cells showing backflow have a limited voltage range. Above that range, the response time is so large that cells become useless in most applications. It has been found that the presence or absence of backflow depends heavily on the pretilt (it is only shown for near-zero pretilts) and on the cell thickness.

Cells showing backflow can be obtained without this effect reducing the cell thickness while keeping constant the remaining manufacturing parameters. When thickness is reduced, backflow either disappears or is shown at higher voltages

\section{Electrooptic Results}

Fig. 3 shows greyscale and dynamic response for $50^{\circ}, 60^{\circ}$, $70^{\circ}$, and $80^{\circ}$ displays.

Cells with $50^{\circ}$ and $60^{\circ}$ evaporation angles have a very low pretilt. They both exhibited backflow when applying voltages greater than $6 \mathrm{~V}$ and showed a double-peaked transmission profile and high response times. For example, rise time was $20 \mathrm{~ms}$ for $10 \mathrm{~V}$. Nevertheless, in a working range up to $6 \mathrm{~V}, 50^{\circ}$ and $60^{\circ}$ displays show very good response time $(3 \mathrm{~ms})$ and excellent contrast ratios.

For $70^{\circ}$ displays, the backflow effect did not show up. As pretilt angle increases, switching becomes easier so turbulences do not appear and backflow effect is avoided. Liquid crystal behavior is standard for this particular angle. Response times are fast in the whole voltage range and faster than for $50^{\circ}$ or $60^{\circ}$. Contrast, however, decreases as a result of the birefringence of the OFF state induced by the pretilt angle.

For $80^{\circ}$ displays, the response shown is similar to $70^{\circ}$. In these cells, however, pretilt is very high (about $26^{\circ}$ ), making the OFF state highly transmissive. Backflow could not be found in these displays either and response times were the fastest due to high pretilt. Rise and fall response times are compared in Table II. Fall times increase as pretilt increases, opposite to rise time. This behavior can be explained by differences in the elastic energy. The higher the pretilt is, the smaller the elastic energy between on and off state becomes and for that reason a longer fall time is expected.

The absence of backflow and the excellent time response should make $70^{\circ}$ and $80^{\circ}$ displays the best choice for applications. However, contrast is poor, thus precluding their use in display applications. This is not the case, however, for photonic 


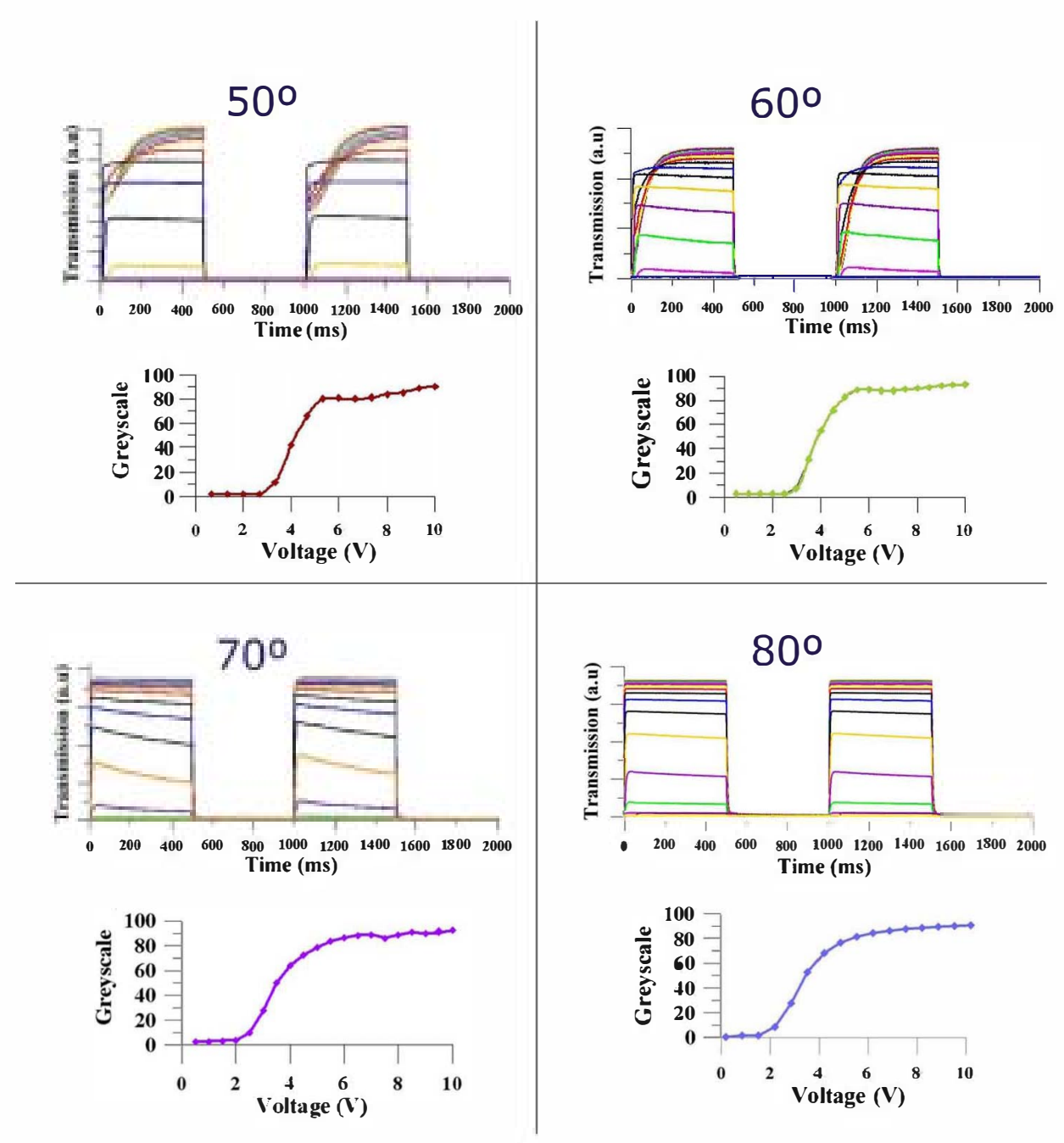

Fig. 3. Electrooptical results for $50^{\circ}, 60,70^{\circ}$ and $80^{\circ}$ displays. Backflow effect can be noticed in $50^{\circ}$ and $60^{\circ}$ displays.

TABLE II

Liquid Crystal Response Times for Various Displays With Different $\mathrm{SiO}_{*:}$ EVAPORATION ANGLES

\begin{tabular}{|c|c|c|c|c|}
\hline SiOx evap. angle & $\mathbf{5 0}^{\mathbf{}}$ & $\mathbf{6 0}^{\circ}$ & $\mathbf{7 0}^{\mathbf{}}$ & $\mathbf{8 0}^{\circ}$ \\
\hline Rise time (ms) & 3.1 & 3.4 & 3.0 & 1.3 \\
\hline Fall time (ms) & 3.5 & 4.8 & 6.6 & 7.5 \\
\hline
\end{tabular}

applications where the variable phase delays of VANs are used. In this case, the only drawback of high pretilt is that the range of delays generated by a given LC material and cell thickness is somewhat reduced. This small disadvantage is superseded in many applications by the excellent dynamic behavior of high pretilt cells.

\section{CONCLUSION}

The results demonstrate, therefore, that $\mathrm{SiO}_{x}$ as aligning surface for VAN LCs may generate a full range of pretilts that can be eventually optimized depending on the requirements of the specific application. In principle, display-related applications should use evaporation angles in the $50^{\circ}-60^{\circ}$ range, so that contrast can be maximized. The contrast requirement shall be met at its minimum for the response time to be improved as much as possible. Non-display applications probably would prefer higher pretilt angles to minimize the response time. The slightly lower phase delay range achieved in these cells should not be relevant for the device performance in most cases

\section{REFERENCES}

[1] J. J. Lyu, J. Sohn, H. Y. Kim, and S. H. Lee, "Recent trends on patterned vertical alignment (PVA) and fringe-field switching (FFS) liquid crystal displays for liquid crystal television applications," J. Display Technol., vol. 3, no. 4, pp. 404-412, Dec. 2007.

[2] W. Shin, T. Won, and C. Lee, "Multi-domain PVA LCD cell: Pros and cons," Mol. Cryst. Liquid Cryst., vol. 476, pp. 433-441, 2007.

[3] S. Choi, J. Hwang, S. Kim, B. Oh, J. Myoung, and D. Seo, "Liquid crystal alignment and electrooptical characteristics of vertical alignment liquid crystal display on $\mathrm{SiO}_{x}$ thin film obliquely deposited by sputtering," Jpn. J. Appl. Phys., vol. 45, pp. 1280-1282, 2006.

[4] C. Chen, P. J. Bos, J. Kim, Q. Li, and J. E. Anderson, "Improved liquid crystals for vertical alignment applications," J. Appl. Phys., vol. 99, pp. 123523-123523, 2006.

[5] P. K. Son, J. H. Park, S. S. Cha, J. C. Kim, and T. H. Yoon, "Vertical alignment of liquid crystal on a-SiO ${ }_{x}$ thin film using the ion beam exposure," Appl. Phys. Lett., vol. 88, pp. 263512-263512, 2006.

[6] P. J. Martin, A. Bendavid, C. Comte, H. Miyata, Y. Asao, Y. Ishida, and A. Sakai, "Alignment and switching behaviors of liquid crystal on a-SiO ${ }_{x}$ thin films deposited by a filtered cathodic arc process," Appl. Phys. Lett., vol. 91, p. 063516. 
[7] X. Quintana, M. A. Geday, B. Cerrolaza, D. Pérez-Medialdea, and J. M. Otón, "Measurement of pretilt angle and cell gap in reflective vertically aligned nematics," in 2007 Spanish Conf. on Electron Devices Proc., 2007, vol. 10, pp. 258-261

[8] A. Marino, E. Santamato, N. Bennis, X. Quintana, J. M. Otón, V. Tkachenko, and G. Abbate, "Ellipsometric study of vertically aligned nematic liquid crystals," Appl. Phys. Lett., vol. 94, pp. 013508-013508, 2009.

[9] S. Kondoh, "Ultra high-resolution FLC display for PDA," Displays, vol. 25, pp. 31-36, 2004.

[10] P. J. M. Vanbrabant and N. D. y. J. F. Strömer, "Temperature influence on the dynamics of vertically aligned liquid crystal displays," Appl. Phys. Lett., vol. 92, pp. 091101-091101, 2008.

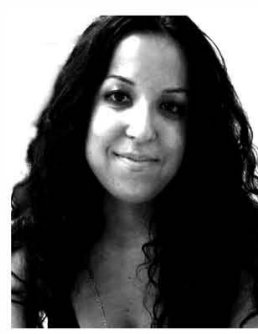

Eva Otón received the B.Sc. degree in chemistry from the Universidad Autónoma de Madrid, in 2005, and the M.Sc. degree in material engineering from the Universidad Complutense de Madrid in 2008.

She joined the Department of Photonic Technology in Universidad Politécnica de Madrid, Madrid, Spain. Her research interest has been focused on vertically aligned nematic liquid crystals and interaction with surface conditioning in $\mathrm{Si} \boldsymbol{\bullet}_{\text {: }}$ and $\mathrm{Si}_{2}$. She is also interested on applications of adaptive optic devices such as liquid crystal graded
Sol López-Andrés, photograph and biography no available at time of publication.

Carlos Carrasco-Vela, photograph and biography no available at time of publication.

Beatriz Cerrolaza, photograph and biography no available at time of publication.

Noureddine Bennis, photograph and biography no available at time of publication.

José Manuel Otón, photograph and biography no available at time of publication. index beam steerers. 